\title{
LASER ASSISTED CHARGE EXCHANGE IN ATOMIC COLLISIONS
}

\author{
J. F. CASTILLO, L. F. ERREA, L. MÉNDEZ and A. RIERA \\ Departamento de Química, CXIV, Universidad Autónoma de Madrid, \\ Cantoblanco, 28049 Madrid, Spain
}

\begin{abstract}
We present total charge exchange cross sections for collisions of $\operatorname{Li}\left(1 \mathrm{~s}^{2} 2 \mathrm{~s}^{2} \mathrm{~S}\right)$ with $\mathrm{H}(1 \mathrm{~s})$ in presence of a linear polarized laser field of intensity $0.05 \leqslant \mathrm{I} \leqslant 1 \mathrm{TW} / \mathrm{cm}^{2}$ and wavelength $510^{3} \leqslant \lambda \leqslant 1410^{3} \AA$. Our calculation shows that the laser field can increase the cross section of this reaction by a factor of ten at impact energies $E<0.1 \mathrm{keV} / \mathrm{a} . \mathrm{m} . \mathrm{u}$. The mechanism of this process is discussed and it is shown that both atomic and molecular radiative transitions can take place depending on the laser wavelength employed.
\end{abstract}

KEY WORDS: Charge exchange, atomic collisions.

In previous papers, ${ }^{1}$ we have suggested that cross sections of charge exchange processes in ion-atom collisions can be increased by inducing radiative transitions between the electronic states of the quasimolecule formed during the collision. Besides that, the presence of the radiation field permits the modification of the cross section by varying two new parameters, intensity and wavelength. This additional freedom makes these processes more selective for diagnosis purposes in fusion plasmas, specially in cold regions of the reactor where the cross sections of the field-free reactions are small.

At the collision velocities considered in this work $(v>0.02$ a.u. $)$, previous calculations have shown that high (although technically accessible ${ }^{2}$ ) laser intensities $\left(\mathrm{I} \simeq 0.1 \mathrm{TW} / \mathrm{cm}^{2}\right)$ are needed to achieve a sizeable effect on the cross sections. The consequence of this requirement is that only a few experiments ${ }^{3}$ have reported cross sections showing the effect of quasimolecular radiative transitions in ion (atom)-atom collisions.

In the present work we have calculated total cross sections for the charge exchange process:

$$
\mathrm{Li}\left(1 \mathrm{~s}^{2} 2 \mathrm{~s}^{2} \mathrm{~S}\right)+\mathrm{H}(1 \mathrm{~s})+\hbar \omega \longrightarrow \mathrm{Li}^{+}+\mathrm{H}^{-}
$$

which has been predicted ${ }^{4}$ to be notably enhanced by the effect of the electromagnetic field. It is known, ${ }^{5}$ that reaction (1) takes place in absence of external fields through non-adiabatic transitions taking place in the (ionic-covalent type) avoided crossings between the electronic molecular states. In this work we have considered the modification of these transitions by a laser frequency that is resonant with the molecular energy splitting at the avoided crossing region. Radiative transitions in pseudocrossings were previously considered ${ }^{6}$ in the $\mathrm{OH}^{8+}$ quasimolecule. Our calculation showed that the dynamical effect of the sharp peak of the transition 
dipole moment is cancelled by the Stark shift of the molecular levels in the avoided crossing region. However investigation is needed to know if these conclusions also hold for wider avoided crossings such as those arising in the LiH correlation diagram.

At the laser intensities considered in the present work, a semiclassical description of the laser is appropriate. In this application we have assumed that the field is monochromatic and linearly polarized. We have also employed a semiclassical description of the collision in which the nuclei follow rectilinear trajectories with impact parameter $\mathrm{b}$ and velocity $\mathrm{v}$, while the electronic motion is described quantummechanically through a wavefunction $\psi(r, t)$, solution of the semiclassical equation:

with $\mathrm{H}$ given by

$$
\left(\mathrm{H}-\left.i \frac{\partial}{\partial t}\right|_{r}\right) \psi=0
$$

$$
\mathrm{H}=\mathrm{H}_{\mathrm{el}}+\mathrm{H}_{\mathrm{int}}
$$

where $\mathrm{H}_{\mathrm{el}}$ is the electronic Hamiltonian in the Born-Oppenheimer approximation and $\mathrm{H}_{\mathrm{int}}$ is the Hamiltonian describing the interaction between the electromagnetic field and the colliding system. In the dipole approximation we have:

$$
\mathrm{H}_{\mathrm{int}}=\sum_{i} \epsilon . \mathbf{r}_{i}
$$

where the sum extends over the number of electrons and

$$
\boldsymbol{\epsilon}=\boldsymbol{\epsilon}_{\mathrm{O}} \cos (\omega t+\phi)
$$

We employ a molecular expansion for $\psi$, in which it is developed in the basis of (approximate) eigenfunctions of $\mathrm{H}_{\mathrm{el}}, \phi_{k}$

$$
\psi(r, t)=\sum_{j} a_{j}(t) \phi(r, \mathrm{R}) \exp \left(-i \int_{0}^{t} E_{k} \mathrm{dt}^{\prime}\right)
$$

where $E_{k}$ is the electronic energy of the molecular state $\chi_{k}$. Substitution of expression (6) in (2) yields the set of differential equations:

$$
i \dot{a}_{k}=\sum_{j} a_{j}\left\langle\phi_{k}\left|\mathrm{H}_{\mathrm{int}}-i \frac{\partial}{\partial t}\right|_{r} \phi_{j}\right\rangle \exp \left[-i \int_{0}^{t}\left(E_{k}-E_{j}\right) \mathrm{dt}^{\prime}\right]
$$

Transition probabilities and cross sections are obtained from the coefficients $a_{k}(t)$ that are a solution of (7). Inspection of (7) shows that non-adiabatic transitions take place through both radiative (due to $\mathrm{H}_{\text {int }}$ ) and non-radiative (due to $\partial / \partial t$ ) couplings. In practice, a perturbative solution ${ }^{7}$ of (7) is usually carried out. In this approximation dynamical couplings are also neglected. A great advantage of the perturbative approximation is that it permits ${ }^{8}$ to obtain a simple expression of the average of the cross section over the orientation of the quasimolecule formed during the collision relative to the direction of polarization of the laser. However, as it will be discussed below, a simple perturbative approach is not adequate for the present calculation. This implies that the system (7) must be solved for each direction of polarization of the laser. In this calculation we have assumed that the laser propagates in the $\mathrm{Y}$ direction of the laboratory frame of reference. This direction corresponds to an electric field $\epsilon$ in the $\mathrm{Z}$ direction, which is expected to yield the largest radiative transitions. 
We have employed a basis set of five molecular states (four ${ }^{1} \Sigma$ and one ${ }^{1} \Pi$ ). The energies of these states ${ }^{5}$ are shown in Figure 1. The entrance channel of reaction (1) is a statistical mixture of $1^{1} \Sigma$ and $1^{3} \Sigma$ (not shown in Figure 1). However, since only one singlet state dissociates in $\mathrm{Li}^{+}+\mathrm{H}^{-}$, in absence of spin-orbit coupling, only the singlet subsystem is needed to treat reaction (1).

The mechanism of the charge exchange process involves a transition between the two first ${ }^{1} \Sigma$ states. As the relative nuclear velocity decreases, the effectiveness of dynamical couplings diminishes, and the $1^{1} \Sigma-2^{1} \Sigma$ transition will take place mainly through radiative transitions. We have selected a range of values of the laser wavelength $\left(510^{3} \leqslant \lambda \leqslant 1410^{3} \AA\right)$ such that radiative transitions from the entrance channel $1^{1} \Sigma$ to states $3^{1} \Sigma$ and $4^{1} \Sigma$ are negligible, therefore the state $4^{1} \Sigma$, which correlates diabatically to $\mathrm{Li}^{+}+\mathrm{H}^{-}$, is populated through an indirect mechanism involving non-radiative transitions in the avoided crossings $2^{1} \Sigma-3^{1} \Sigma$ and $3^{1} \Sigma-4^{1} \Sigma$. A practical consequence of this mechanism, which involves both radiative and non-radiative couplings, is that a perturbative approximation is not adequate for the present calculation.

We show in Figure 2 the transition dipole moments that are relevant to the mechanism proposed. One can notice that the transition dipole moments $\left\langle 1^{1} \Sigma|\mu| 2^{1} \Sigma\right\rangle$ and $\left\langle 1^{1} \Sigma|\mu| 1^{1} \Pi\right\rangle$ do not vanish asymptotically. To see the implications of this fact, we have solved (7) assuming that the colliding system is described initially by the atomic state $\operatorname{Li}\left(1 s^{2} 2 s^{2} s\right)$. We have found that even for laser frequencies not

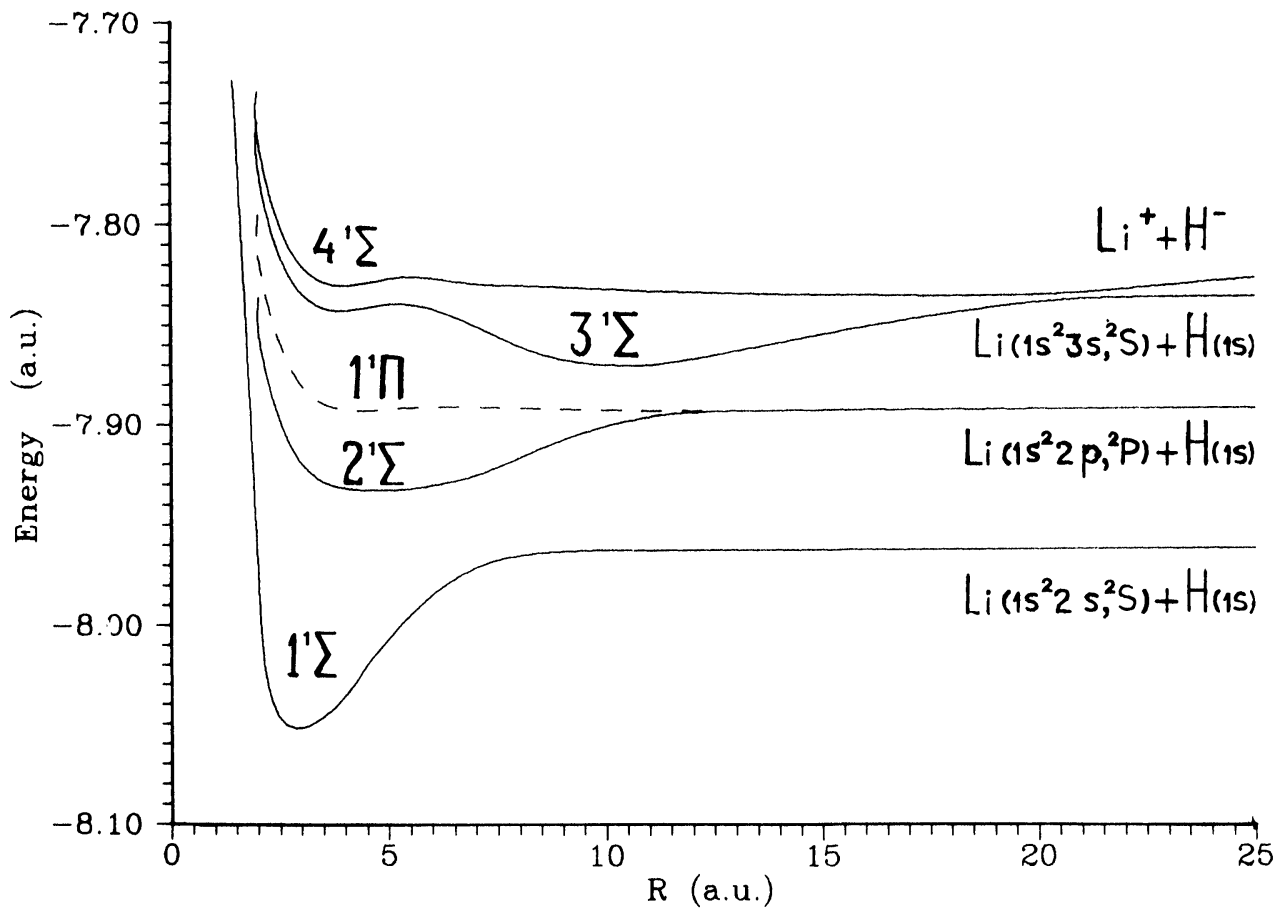

Figure 1 Electronic energies of the states ${ }^{1} \Sigma(----)$ and ${ }^{1} \Pi$ of LiH. 


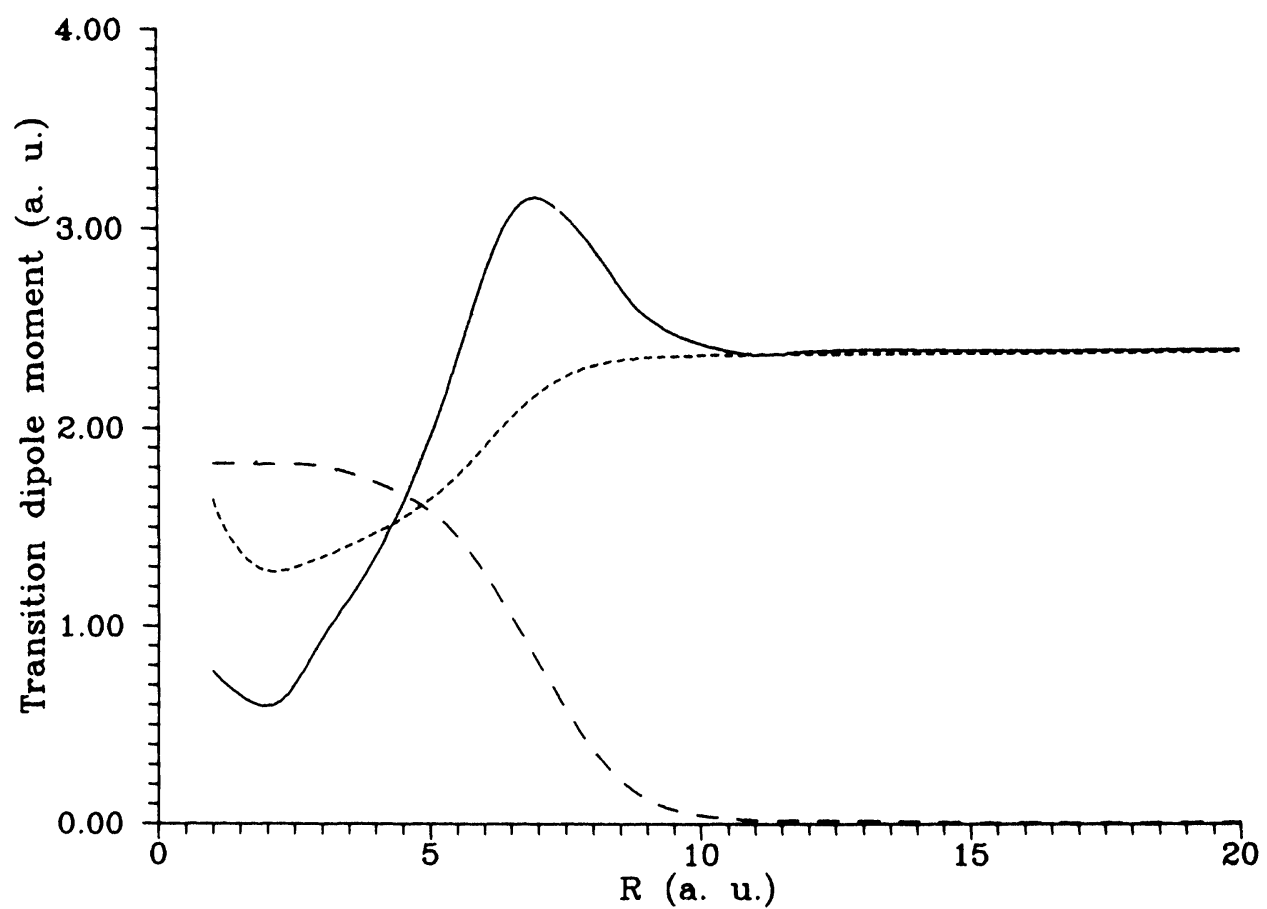

Figure 2 Modulus of transition dipole moments as functions of the internuclear distance: (- $\longrightarrow$ ) $\left.k 1^{1} \Sigma|\mu| 2^{1} \Sigma\right\rangle|;(----)|\left\langle 1^{1} \Sigma|\mu| 1^{1} \Pi\right\rangle|;(----)|\left\langle 2^{1} \Sigma|\mu| 1^{1} \Pi\right\rangle \mid$.

satisfying the resonance condition in the atomic limit $(\omega \simeq 0.07$ a.u. $)$, the coefficients $a_{k}(t)$ oscillate indefinitely reflecting the inadequacy of this approximate initial condition. To overcome this difficulty, we have solved the system (7) assuming that the $\mathrm{Li}$ atom is described initially by a linear combination of $\mathrm{Li}\left(1 \mathrm{~s}^{2} 2 \mathrm{~s}^{2} \mathrm{~S}\right)$ and $\mathrm{Li}\left(1 \mathrm{~s}^{2} 2 \mathrm{~s}{ }^{2} \mathrm{P}\right)$ atomic states, an atomic "dressed" state, ${ }^{9}$ formed by interaction with the laser field. Physically this initial condition implies that the time of interaction of the $\mathrm{Li}$ atom with the field is longer than the time of interaction with the $\mathrm{H}$ atom. In practice we have assumed that the system is initially described for the dressed state with a larger contribution of the atomic state $\operatorname{Li}\left(1 \mathrm{~s}^{2} 2 \mathrm{~s}^{2} \mathrm{~S}\right)$. To implement the new initial condition, we have transformed the molecular basis set to obtain a set of wavefunctions which dissociate in the atomic dressed states. The new basis set is formed by the following functions:

$\phi_{1}^{d}=c_{1}^{+} \phi_{1 \Sigma} \exp \left(-i E_{1}^{0} t\right)+c_{2}^{+}\left(\cos \theta \phi_{2 \Sigma}+\sin \theta \phi_{1 \Pi}\right) \exp \left(-i E_{2}^{0} t\right)$

$\phi_{2}{ }^{d}=c_{1}-\phi_{1 \Sigma} \exp \left(-i E_{1}{ }^{0} t\right)+c_{2}-\left(\cos \theta \phi_{2 \Sigma}+\sin \theta \phi_{1} \Pi\right) \exp \left(-i E_{2}{ }^{0} t\right)$

$\phi_{3}{ }^{d}=\left(-\sin \theta \phi_{2 \Sigma}+\cos \theta \phi_{1 \Pi}\right) \exp \left(-i E_{2}{ }^{0} t\right)$

$\phi_{4}{ }^{d}=\phi_{3 \Sigma}$

$\phi_{5}{ }^{d}=\phi_{4 \Sigma}$ 
where the coefficients $c^{ \pm}$are those defining the dressed states, ${ }^{9} E_{1,2}{ }^{\circ}$ are the energies of the atomic states $\operatorname{Li}\left(1 \mathrm{~s}^{2} 2 \mathrm{~s}\right)$ and $\operatorname{Li}\left(1 \mathrm{~s}^{2} 2 \mathrm{p}\right)$, and $\sin \theta=\mathrm{b} / \mathrm{R}, \cos \theta=v t / \mathrm{R}$ are the elements of the matrix that asymptotically transforms molecular wavefunctions into non-rotating ones. Substitution of the new expansion in (2) yields an alternative set of differential equations. Charge exchange cross sections are obtained in our basis set from the coefficient of $\chi_{s}^{d}$.

Charge exchange cross sections are presented in Figures 3 and 4 as functions of laser wavelength and impact energy. It must be noted that, since the coefficients $c_{i}^{+}$ are oscillating functions of $t$, the calculated cross section depends on the choice of the time origin. As a consequence, the cross section oscillates as a function of the phase of the field $\phi$ (values given in Figures 3 and 4 are for $\phi=0$ ). The amplitude of this oscillation is about one third of the cross section mean value.

The calculated cross section (Figure 3 ) shows two maxima as a function of the laser wavelength. The first maximum at $\lambda \simeq 9700 \AA$ appears at a laser frequency that corresponds to the $\operatorname{Li}\left(1 s^{2} 2 p\right)-\operatorname{Li}\left(1 s^{2} 2 s\right)$ energy difference, and this peak is due to an enhancement of the cross section due to radiative transitions taking place in the $\mathrm{Li}$ atom before entering in the collision region. The second maximum appears at $\lambda \simeq 6600 \AA$, which corresponds to the $2^{1} \Sigma-1^{1} \Sigma$ energy difference at $\mathrm{R} \simeq 7 \mathrm{Bohr}$, and may be ascribed to quasimolecular radiative transitions in the vicinity of the $2^{1} \Sigma-1^{1} \Sigma$ avoided crossing, which in contrast with previous findings are not

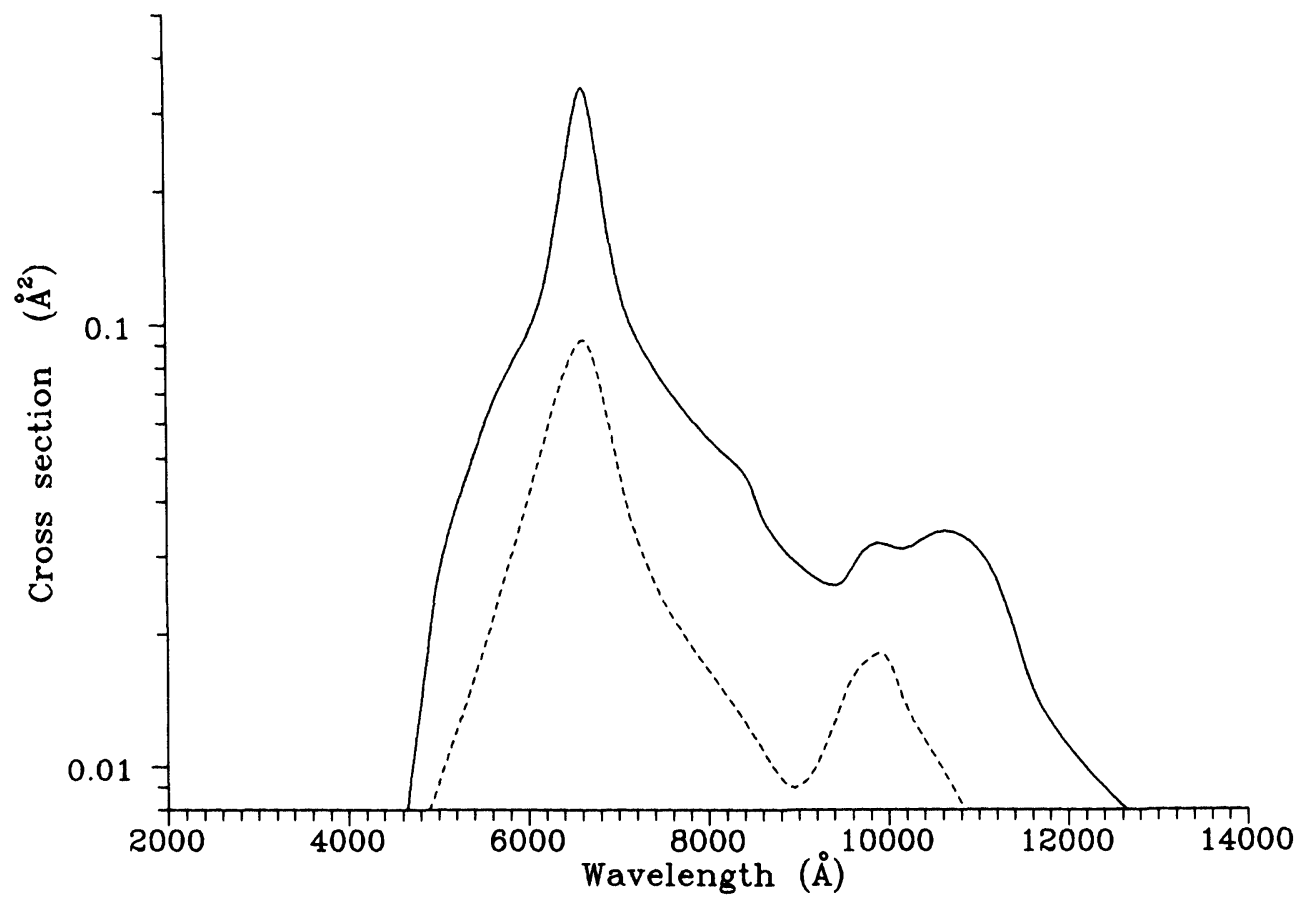

Figure 3 Charge exchange total cross section as a function of the laser wavelength for two impact energies: $(\longrightarrow) E=30 \mathrm{eV} /$ a.m.u.; $(----) E=40 \mathrm{eV} / \mathrm{a} . \mathrm{m}$. u., and laser intensity $\mathrm{I}=0.1 \mathrm{~T} \mathrm{w} / \mathrm{cm}^{2}$. 


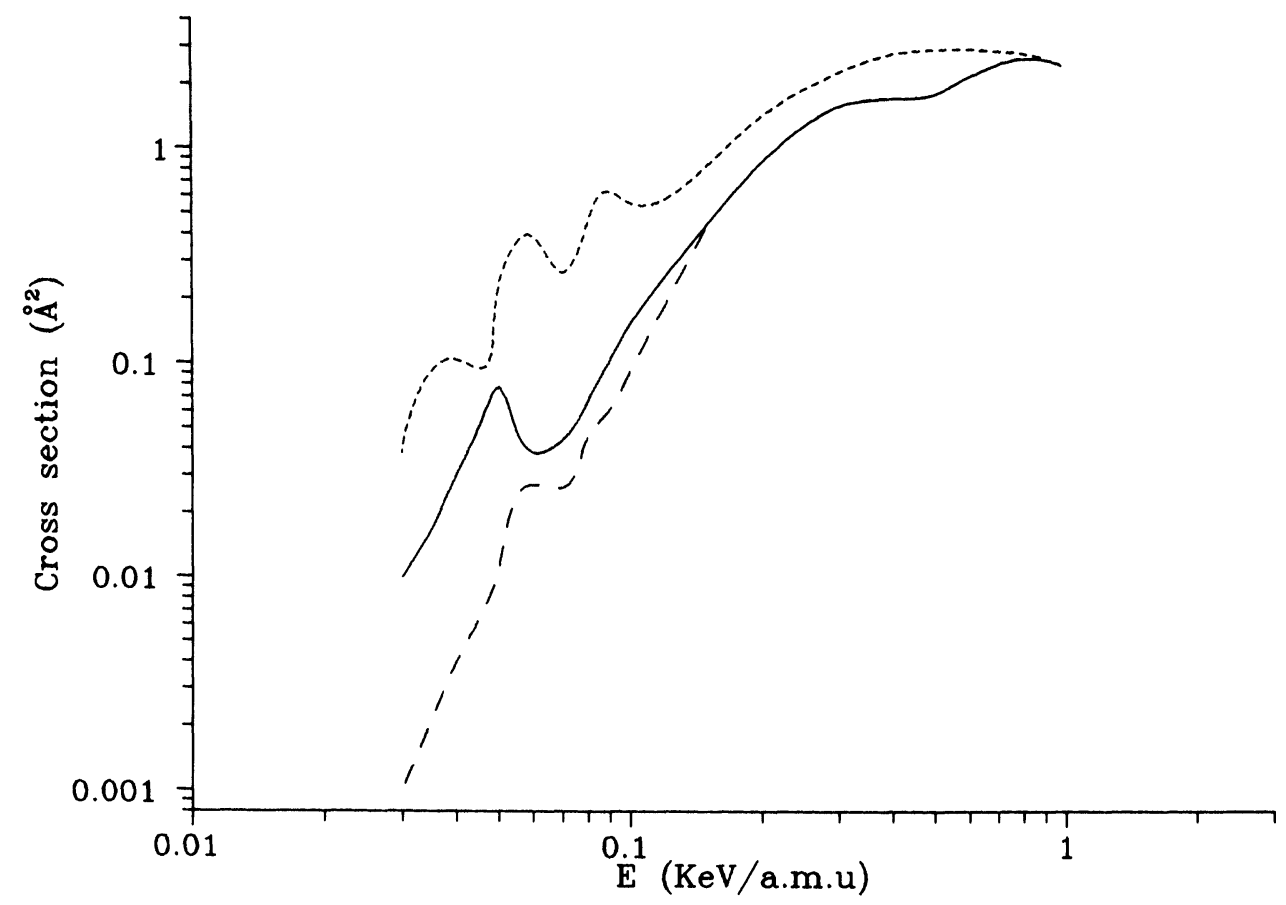

Figure 4 Charge exchange total cross section as a function of the impact energy for two laser

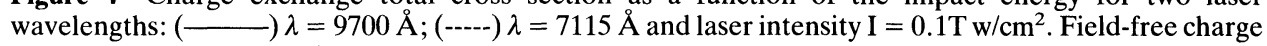
exchange total cross sections.

completely cancelled out by the diagonal Stark terms. A similar behaviour has been found for other values of the impact energy and laser intensity.

In Figure 4 we present the dependence of the charge exchange cross section with the impact energy. It can be noted that the cross section is enhanced in the region of impact energies $E<0.1 \mathrm{keV} / \mathrm{a} . \mathrm{m} . \mathrm{u}$. where dynamical transitions cease to be effective. Quantal calculations at lower velocities, where the effect of radiative transitions can be more pronounced, are underway.

\section{References}

1. L. F. Errea, L. Méndez and A. Riera, J. Phys. B 18, 4271 (1985) and references therein.

2. C. K. Rhodes, Science 229, 1435 (1985).

3. W. R. Green, M. D. Wright, J. P. Young and S. E. Harris, Phys. Rev. Lett. 43, 120 (1979); A. Debarre and Ph. Cahuzac, J. Phys. B 19, 3965 (1986); M.

4. A. M. F. Lau, Phys. Rev. A 13, 139 (1976).

5. L. Méndez, I. L. Cooper, A. S. Dickinson, O. Mó and A. Riera, J. Phys. B 23, 2797 (1990).

6. L. F. Errea, L. Méndez and A. Riera, J. Chem. Phys. 85, 825 (1988).

7. D. A. Copeland and C. L. Tang, J. Chem. Phys. 65, 3161 (1976).

8. L. F. Errea, L. Méndez and A. Riera, J. Chem. Phys. 49, 4221 (1983).

9. M. H. Mittleman Theory of Laser-Atom Interaction, Plenum Press, New York (1982). 